\title{
Stromal cell-derived factor 1 secreted by cancer- associated fibroblasts promotes IncRNA Xist through CXCR4 and facilitates invasion and metastasis of non-small cell lung cancer
}

\author{
Lifang Wang \\ school of basic medicine and forensic medicine ,hangzhou medical college \\ Wenhu Chen \\ Hangzhou Medical College \\ Xiaoyan Li \\ Hangzhou Medical College \\ Hongguang Zhao ( $\sim$ Hongguang11072@163.com ) \\ Zhejiang Cancer Hospital https://orcid.org/0000-0002-8278-6080
}

\section{Research}

Keywords: Non-small cell lung cancer, Cancer-associated fibroblasts, LncRNA XIST, miR-15a-5p, MMP3, Stromal cell-derived factor 1, CXCR4

Posted Date: May 10th, 2021

DOI: https://doi.org/10.21203/rs.3.rs-500633/v1

License: (1) This work is licensed under a Creative Commons Attribution 4.0 International License. Read Full License

Version of Record: A version of this preprint was published at Current Cancer Drug Targets on April 7th, 2022. See the published version at https://doi.org/10.2174/1568009622666220407102725. 


\section{Abstract}

Cancer-associated fibroblasts (CAFs) abundantly exist in NSCLCs and promote tumor progression in most solid tumors. This study investigated the effect of CAFs on NSCLC. CAFs and normal fibroblasts (NFs) were isolated and identified. CAFs or NFs were cocultured with NSCLCs cells. Cell invasion and migration, and expression of E-cadherin, N-cadherin, SDF-1, CXCR4 and Xist were measured. SDF1/CXCR4 axis inhibitor ADM3100 or low expression of Xist was added to verify the invasion and metastasis of NSCLC. The binding relationships of Xist, miR-15a-5p and MMP3 were analyzed. The effect of miR-15a-5p overexpression on H1299 was evaluated. Finally, lung metastasis experiment was carried out in vivo. CAFs promoted the invasion and EMT of NSCLC cells by secreting SDF-1. CAFs increased CXCR4 and Xist expressions in NSCLC cells. SDF-1 knockdown or ADM3100 decreased CXCR4 and Xist expressions. Silencing Xist reduced the promotion of CAFs on NSCLC. Xist targeted miR-15a-5p to regulate MMP3. Overexpression of miR-15a-5p promoted the invasion and EMT of NSCLC cells. CAFs caused more metastatic nodules and elevated SDF-1, CXCR4, MMP3 and XIST levels, and lowered miR15a-5p level. Collectively, SDF-1 secreted by CAFs promotes IncRNA Xist through CXCR4, and Xist targets miR-15a-5p to regulate MMP3, thus promoting invasion and metastasis of NSCLC.

\section{Introduction}

Lung cancer (LC) is the primary cancer killer worldwide, which imposes grave challenges for patients and clinicians [1]. The most common histology is non-small cell lung cancers (NSCLC), which accounts for nearly $85 \%$ of cases [2], and the vast majority of NSCLC patients are found in in the stage of advanced inoperable[3] since early stage tumor development is usually asymptomatic [4]. The prognosis of early NSCLC is poor even if it is treated by surgery and neoadjuvant chemotherapy [5]. Up to 70\% of NSCLC patients develop central nervous system metastases during the course of their disease [6]. The median overall survival rate of advanced NSCLC patients is approximately 10 months[7]. Unfortunately, the clinical response is almost temporary and acquired resistance to these drugs invariably emerges [8]. Therefore, it is of great clinical significance to better understand molecular mechanism and further search for effective and updated comprehensive options for diagnosis and treatment and minimizing related risks.

Cancer-associated fibroblasts (CAFs) are most important host cells in tumor microenvironment, and some studies have found that CAFs are well-known to promote tumor angiogenesis and the malignant progression of cancer $[9,10]$. CAFs are the most common component of non-tumor cells in most solid tumors, which are important in immunotherapy resistance by significantly affecting tumor neogenesis, persistence and metastasis [11]. CAFs are associated with increased malignant potential and the acquisition of stem cell-like properties in NSCLC, and certainly promote epithelial-mesenchymal transition (EMT) and metastasis in NSCLC $[12,13]$.

In addition, CAFs can secrete typical fibroblast products and affect extracellular matrix formation and remodeling, and uniquely secrete factors to promote cancer cell dissemination [14]. A higher amount of 
stromal cell-derived factor 1 (SDF-1) exists in the CAFs rather than that in the normal fibroblasts (NFs) [15]. SDF-1, also known as Chemokine 12 (CXCL12) is ubiquitously expressed in many tissues and cell types, and it can specifically interact with the ligand for CXCR4 [16]. In clinical LC samples, CXCR4 is highly expressed in both normal and tumor tissues [17]. The CXCL12/SDF-1 and its receptor CXCR4 play a major role in tumor initiation, promotion, progression and metastasis $[12,13,18]$. CXCR4 and CXCL12 knockdown is conducive to inhibiting NSCLC growth and proliferation, migration and invasion of A549 cells [19]. However, there is little report on the mechanism of CAF-secreted SDF-1 in NSCLC development with the regulation of CXCR4. This paper aims to evaluate the specific mechanism of CAFs on NSCLC and hopes to provide theoretical and experimental basis for NSCLC management.

\section{Materials And Methods}

\section{Ethics statement}

All procedures were authorized by the academic ethics committee of Cancer Hospital Affiliated to the University of Chinese Academy of Sciences. The experiment was carried out in strict accordance with the guidelines for the management and use of laboratory animals. All the laboratory procedures were used to reduce the pain of the mice.

\section{Isolation and cultured of CAFs and NFs}

CAFs and NFs were isolated from cancer tissues and adjacent normal lung tissues of 10 patients (7 males and 3 females, mean age 69 years) with NSCLC. The inclusion criteria were patients with primary NSCLC whose tumor stage was IA to IVA. Surgery was used as the initial treatment without preoperative chemotherapy or radiotherapy. The patients with other diseases or tumors were excluded. Lung cancer and normal lung tissue were cut into $1 \mathrm{~mm}^{3}$ small pieces and cultured in DMEM (Nacalai Tesque Inc., Kyoto, Japan) containing 10\% fetal bovine serum (FBS) (GIBCO, Grand Island, NY, USA), $100 \mathrm{U} / \mathrm{mL}$ penicillin and $100 \mu \mathrm{g} / \mathrm{mL}$ streptomycin, respectively. The medium was changed once a week until the tissue was surrounded by adherent fibroblasts. After about 3 weeks, fibroblasts were separated from epithelial cells and endothelial cells by trypsin. When the confluence reached $80 \%$, the cells were passaged at the ratio of 1:3. The morphology of CAFs and NFs was observed under the inverted microscope (CKX41, Olympus, Tokyo, Japan).

\section{Immunofluorescence staining}

CAFs and NFs were seeded on 24-well plates. After 24 hours, they were fixed with $4 \%$ paraformaldehyde for 30 minutes, infiltrated with $0.3 \%$ Triton X-100 for 5 minutes, and incubated with antibodies against fibroblast activation protein-a (FAPa) (ab28244, Abcam, Cambridge, MA, USA) and a-smooth muscle actin (a-SMA) (ab124964, Abcam) overnight at $4^{\circ} \mathrm{C}$. After 3 regimens of with phosphate-buffered saline (PBS) washing, secondary goat anti-rabbit IgG H\&L (FITC) (ab6717, Abcam) was used for incubation at room temperature for 2 hours. Finally, the cover glass was mounted on the slide and examined under the inverted fluorescence microscope (TS100-F, Nikon, Tokyo, Japan). 


\section{Culture of A549 and H1299 cells}

Human NSCLC cells A549 and H1299 (ATCC, Manassas, VA, USA) were cultured in RPMI 1640 (GIBCO) containing $10 \%$ fetal bovine serum, $50 \mathrm{U} / \mathrm{mL}$ penicillin and $50 \mathrm{U} / \mathrm{mL}$ streptomycin. NSCLC cells were cocultured with CAFs or NFs at a ratio of $5: 1$, and the total number of cells was $1 \times 10^{6}$.

\section{Cell grouping}

The construction and packaging of SDF-1 interference vector si-SDF-1 and control si-NC, IncRNA XIST interference vector si-XIST and control Xist NC, miR-15a-5p mimic and miR-NC were carried out by Gene Pharma (Shanghai, China). Lipofectamine 3000 (Thermo Fisher Scientific) was used for transfection according to the instructions. AMD3100 (Catalog No. CAS 155148-31-5) was purchased from Santa Cruz biotechnology, Inc. (Dallas, TX, USA) and dissolved in dimethyl sulphoxide (DMSO) at $2 \mu \mathrm{mol} / \mathrm{L}$. The following experiment was carried out 48 hours later.

Cells were assigned into NFs, CAFs, CAFs-si-NC, CAFs-si-SDF-1, CAFs + DMSO, CAFs + AMD3100, CAFs + si-NC, CAFs + si-XIST, CAFs + miR-NC and CAFs + miR-15a-5p groups.

\section{Transwell assay}

All $1 \times 10^{5}$ cells were seeded in serum-free medium in the apical chamber of 24-well Transwell (8- $\mu \mathrm{m}$ pore size, Corning Costar) pretreated with Matrigel (BD Biosciences, Franklin Lakes, NJ, USA), and the medium containing 10\% FBS was added in the basolateral layer. The cells were cultured for 24 hours, stained with $0.1 \%$ crystal violet solution, observed and counted under the microscope (Olympus).

\section{Scratch test}

When the confluence reached $90 \%$, the cells were seeded in 6-well plates, cultured for 24 hours, and scratched vertically on the cell surface with $200 \mu \mathrm{L}$ sterile pipette. The cells were cultured in serum-free medium and photographed with Image Pro Plus software (Media Cybernetics, Rockville, MD, USA) at different time points ( 0 and 24 hours).

\section{Dual-luciferase experiment}

The binding sequence and mutation sequence of IncRNA XIST and miR-15a-5p, miR-15a-5p and MMP3 were cloned into psicheck2 luciferase vector (Promega, Madison, WI, USA) to construct wild-type plasmids XIST-WT, MMP3-WT and mutant plasmids XIST-MUT and MMP3-MUT. The constructed plasmids were cotransfected with mimic NC and miR-15a-5p mimic into HEK293T cells (ATCC). Lipofectamine 2000 (Life Technologies) was used as the transfection reagent. The luciferase activity was detected 48 hours later.

\section{RNA immunoprecipitaion (RIP) assay}

EZ Magna rip Kit (Millipore, Bedford, MA, USA) was used for RIP analysis. Cells were collected and resuspended with RIP lysate. Then, it was incubated with RIP buffer containing magnetic beads and 
human anti-Ago2 antibody (Millipore). The precipitates were digested with proteinase $\mathrm{K}$, and then RNA was isolated and purified, and analyzed by qPCR.

\section{Metastatic model of rat NSCLC in nude mice}

A total of 12 4-week-old male BALB/C nude mice weighing 13-15g were purchased from Beijing Vital River Laboratory Animal Technology Co., Ltd [Beijing, China, license number: SYXK (Beijing) 2017-0033].

Totally $2 \times 10^{6}$ cells were injected into the tail vein of nude mice. Four weeks later, the mice were killed. The number of metastatic nodules on the surface of lung was calculated by hand. Hematoxylin and eosin (HE) staining was used for histological analysis. The animals were randomly divided into $\mathrm{H} 1299+$ NFs group and H1299 + CAFs group.

\section{HE staining}

The lung tissue was paraffin embedded, sliced at $5 \mu \mathrm{m}$, dewaxed with xylene and dehydrated with ethanol, dyed with hematoxylin for $5 \mathrm{~min}$, and soaked with double distilled water. Next, the sections were dyed with eosin for $2 \mathrm{~min}$, soaked with xylene for $2 \mathrm{~min}$, sealed with neutral resin and observed under light microscope.

\section{Reverse transcription quantitative polymerase chain reaction (RT-qPCR)}

The TRIzol (Invitrogen, Carlsbad, CA, USA) was used to extract the total RNA. According to the instructions, the total mRNA was reverse transcribed using the miScript reverse transcription kit (Qiagen, Valencia, CA, USA), and then the RNA expression was detected using the MiScript SYBR Green PCR kit (Qiagen), with U6 as the internal reference. For mRNA expression, reverse transcription was performed with SuperScript III First-Strand Synthesis System (Invitrogen), and RT-qPCR was performed with Powerup SYBR Green qPCR master mix in an ABI 7500 fast real-time PCR system (Applied Biosystems, Carlsbad, $C A$, USA). GAPDH was used as internal reference. The relative expression was expressed by $2^{-\triangle \Delta C T}$ [20]. The primer sequences are listed in Table 1. 
Table 1

Primer sequences

\begin{tabular}{|lll|}
\hline Gene & Forward 5'-3' $^{\prime}$ & Reverse 5'-3' $^{\prime}$ \\
\hline FAP-a & atgaagacttgggtaaaaat & atctccaaagcatggttcta \\
\hline a-SMA & atgtgtgaagaagaggacag & agtcattgtagaaagagtgg \\
\hline SDF-1 & atgaacgccaaggtcgtggt & tcacatcttgaacctcttgt \\
\hline CXCR4 & atgtccattcctttgcctct & tcatgcttctcagtttcttc \\
\hline LncRNA XIST & TGCTGATCATTTGGTGGTGT & TGACTTCCTCTGCCTGACCT \\
miR-15a-5p & TAGCAGCACATAATGG & CAGTGCGTGTCGTGGA \\
\hline MMP3 & atgaagagtcttccaatcct & atcagcctctccttcataca \\
\hline U6 & AGACCGTTCGTCAACCTAGC & GAAAGACCGCAGCAAAATTC \\
\hline GAPDH & atggtgaaggtcggtgtgaa & agttgtcattgagagcaatg \\
\hline
\end{tabular}

\section{Western blot analysis}

RIPA (Beyotime, Shanghai, China) was used to extract the total protein of cells or tissues, and bicinchoninic acid method was used to detect the protein concentration. 15\% SDS-PAGE electrophoresis was used. After successful membrane transfer, $3 \%$ skim milk was used to block the membranes for 2 hours, and PBST was used to clean the membranes. Then, primary antibodies SDF-1 (ab25117, Abcam), CXCR4 (ab181020, Abcam), matrix metalloproteinase 3 (MMP3) (ab52915, Abcam), E-cadherin (ab76011, Abcam) and N-cadherin (ab76319, Abcam) were added and incubated overnight at $4^{\circ} \mathrm{C}$. After 90 min incubation with the secondary antibody goat anti-rabbit IgG H\&L (HRP) (ab205718, Abcam), the membranes were washed with PBS, developed with chemiluminescence, and gray value was analyzed. The internal reference was GAPDH (ab9485, Abcam).

\section{Statistical analysis}

GraphPad Prism 8.01 (GraphPad Software Inc., San Diego, CA, USA) was used for data analysis and mapping. Data were in normal distribution and expressed as mean \pm standard deviation. The $t$ test was used for group pair comparison. One-way analysis of variance (ANOVA) and two-way ANOVA were used for comparison among groups. Tukey's or Sidak's multiple comparisons test was used for post hoc test. $P<0.05$ indicated statistical significance.

\section{Results}

\section{Identification of CAFs}

NSCLC is the main cause of cancer death and the survival rate is less than $5 \%$, and the prognosis is poor[21, 22]. CAFs have been found in NSCLC, which can promote tumor progression and metastasis [15]. 
In order to study the effect of CAFs on NSCLC, we first isolated and cultured CAFs and NFs from NSCLC tissues. Under the microscope, CAFs were spindle or long fusiform, with different sizes, disorderly arrangement and overlapping growth, while NFs were mostly flat stellate with consistent sizes, orderly arrangement in a certain direction and without overlapping growth (Fig. 1A). Further immunofluorescence staining of FAP- $a$ and $a-S M A$ showed that the protein contents of FAP- $a$ and a-SMA in CAFs were higher than those in NFs (Fig. 1B). RT-qPCR showed that the mRNA expressions of FAP-a and a-SMA in CAFs were higher than those in NFs (Fig. 1C, all $p<0.01$ ).

\section{CAFs promote invasion and metastasis of NSCLC}

In order to study the effect of CAFs on the invasion and metastasis of NSCLC, Transwell assay and scratch test were used to detect the changes of invasion and migration ability of cells cocultured with CAFs and A549 or $\mathrm{H} 1299$ cells. The results showed that CAFs improved the invasion and migration ability of A549 or H1299 cells (Fig. 2A-B, all $p<0.01$ ). Local progression and metastasis of NSCLC are related to a variety of mechanisms, especially EMT; through EMT, cancer cells can obtain stronger migration and invasion ability $[23,24]$. Therefore, we detected the protein levels of E-cadherin and N-cadherin by Western blot, and found that CAFs reduced E-cadherin expression and increased N-cadherin (Fig. 2C, all $p<0.01$ ). These results suggest that CAFs can promote the invasion and metastasis of NSCLC.

\section{SDF-1 secreted by CAFs promote invasion and metastasis of NSCLC}

CAFs can secrete a variety of soluble factors including SDF-1 through paracrine action, which can promote tumor invasion and metastasis [15]. Therefore, in order to study the effect of SDF-1 in CAFs on NSCLC, we detected SDF-1 expression by RT-qPCR, which showed that SDF-1 was highly expressed in CAFs (Fig. 3A, all $p<0.01$ ). After CAFs were cocultured with A549 or H1299 cells, SDF-1 expression in A549 and H1299 cells was also increased (Fig. 3B, p < 0.01). We transfected si-SDF-1 into CAFs, and compared with si-NC, we successfully reduced the expression of SDF-1 in CAFs (Fig. 3C, all $p<0.01$ ). After coculture of CAFs low-expressing SDF-1 with A549 or H1299 cells, SDF-1 expression in A549 and H1299 cells was also decreased (Fig. 3D, P < 0.01). Compared with CAFs-si-NC group, CAFs-si-SDF-1 group had reduced invasion and migration (Fig. 3E-F), elevated expression of E-cadherin and lowered Ncadherin (Fig. 3G, all $p<0.01$ ). Briefly, SDF-1 secreted by CAFs can promote the invasion and metastasis of NSCLC.

\section{SDF-1 promotes IncRNA Xist expression through CXCR4}

SDF-1 has high affinity for CXCR4, a specific receptor on the surface of tumor cells. SDF-1 is important in tumor metastasis and invasion by activating CXCR4 [15]. The activation of SDF-1/CXCR4 axis can upregulate IncRNA Xist and participate in the occurrence and development of rectal cancer [25]. LncRNA Xist is involved in the intimal metastasis and invasion of NSCLC $[26,27]$. Therefore, we speculated whether SDF-1 promotes NSCLC by regulating Xist through CXCR4. RT-qPCR demonstrated that CAFs 
increased the levels of CXCR4 and Xist in A549 or H1299 cells, while silencing SDF-1 in CAFs decreased the expression of CXCR4 and Xist. Then, we added AMD3100, an inhibitor of CXCR4, to CAFs group. Compared with CAFs + DMSO group, the expressions of CXCR4 and Xist in CAFs + AMD3100 group were decreased (Fig. 4A-B, all $p<0.01$ ). These results suggest that SDF-1 can promote the expression of Xist through CXCR4.

\section{Silencing IncRNA Xist reduces the promoting effect of SDF- 1 secreted by CAFs on NSCLC}

To further study the promoting effect of Xist on NSCLC, we transferred si-Xist to H1299 cells, with si-NC as control. After successfully reducing Xist expression (Fig. 5A, p < 0.01), we cocultured H1299 cells with CAFs and found that Xist in CAFs + si-Xist group was also significantly decreased (Fig. 5B, $p<0.01$ ). Then the ability of invasion and metastasis was detected. Compared with CAFs + si-NC group, the invasion and migration of cells in CAFs + si-Xist group were decreased (Fig. 5C-D, all $p<0.01$ ). Silencing Xist increased $\mathrm{E}$-cadherin and reduced $\mathrm{N}$-cadherin levels (Fig. $5 \mathrm{E}$, all $p<0.01$ ). Overall, silencing Xist can reduce the promoting effect of SDF-1 secreted by CAFs on the invasion and metastasis of NSCLC.

\section{LncRNA Xist targets miR-15a-5p to regulate MMP3}

To further study the specific molecular mechanism of IncRNA Xist in the tumorigenesis of NSCLC, we used Starbase (http://starbase.sysu.edu.cn/index.php) [28] to predict the downstream miRNAs (Fig. 6A). Among them, miR-15a-5p is associated with the pathogenesis of NSCLC and is low expressed in the serum of patients with NSCLC[29]. Then we further predicted the downstream target genes of miR-15a-5p (Fig. 6B), in which MMP3 affects the migration and invasion of NSCLC[30, 31]. These results implied that IncRNA Xist may monitor NSCLC progression through miR-15a-5p/MMP3. We confirmed the binding relationships between Xist and miR-15a-5p, and miR-15a-5p and MMP3 through the obtained binding sites, luciferase-reporter assay and RIP assay (Fig. 6C-D, all $p<0.01$ ). RT-qPCR showed that CAFs reduced miR-15a-5p and increased MMP3, while silencing SDF-1 or AMD3100 or silencing Xist could increase miR-15a-5p and decrease MMP3 (Fig. 6E, all $p<0.01$ ). In short, IncRNA Xist can target miR-15a-5p to regulate MMP3.

\section{Overexpression of miR-15a-5p partially reverses the promotion of SDF-1 secreted by CAFs on NSCLC}

To study the effect of miR-15a-5p on NSCLC, we transfected miR-15a-5p mimic into H1299 cells, with miR-NC as control (Fig. 7A, $p<0.01$ ). After coculture with CAFs, miR-15a-5p was also upregulated in CAFs + mimic group (Fig. 7B, $p$ < 0.01). Compared with CAFs + miR-NC group, the invasion and migration of CAFs + mimic group were decreased (Fig. 7C-D, all $p<0.01$ ). Overexpression of miR-15a-5p increased Ecadherin and decreased N-cadherin levels (Fig. 7E) and reduced MMP3 expression (Fig. 7F, all $p<0.01$ ). 

NSCLC.

\section{SDF-1 secreted by CAFs promotes lung metastasis through CXCR4}

To study the role of SDF-1 secreted by CAFs in lung cancer metastasis in vivo, we cocultured CAFs and H1299 cells and injected them into mice. Compared with H1299 + NFs group, H1299 + CAFs group had more metastatic nodules on the lung surface (Fig. 8A-B, p < 0.01). Compared with H1299 + NFs group, Xist was upregulated, miR-15a-5p was downregulated (Fig. 8C), E-cadherin was downregulated, and SDF-1, CXCR4, MMP3 and N-cadherin were upregulated in H1299+CAFs group (Fig. 8D, all $p<0.01$ ). These results suggest that SDF-1 secreted by CAFs can affect Xist/miR-15a-5p/MMP3 through CXCR4 and promote lung metastasis in nude mice.

\section{Discussion}

This study initially supported that SDF- 1 secreted by CAFs promoted IncRNA Xist expression by binding to CXCR4, and then lowered miR-15a-5p and enhanced MMP3 to stimulated NSCLC progression.

The crosstalk between malignant cells and CAFs actively contributes to tumor growth and metastatic dissemination [32,33]. Eliminating CAFs or reducing their tumor-promoting activity is beneficial for tumor immunotherapy [34]. Through EMT, cancer cells can obtain stronger migration and invasion ability [23, 24]. In this study, CAFs and NFs were isolated from NSCLC tissues. Immunofluorescence staining and RTqPCR showed that the protein contents of FAP-a and a-SMA in CAFs were higher than those in NFs. After CAFs were cocultured with A549 or H1299 cells, the invasion and migration ability of A549 or H1299 cells were improved, E-cadherin expression was reduced and N-cadherin was increased. CAFs underwent increased aerobic glycolysis and promoted the EMT, migration, and invasion of NSCLC cells [35]. These results suggest that CAFs can promote the invasion and EMT of NSCLC.

CAFs can secrete SDF-1 through paracrine action, which can promote tumor invasion and metastasis [15]. RT-qPCR showed SDF-1 was highly expressed in CAFs and A549 or H1299 cells cocultured with CAFs. Consistently, lung adenocarcinomas overexpressed CXCR4 and CXCL12 [36]. We then transfected si-SDF-1 into CAFs, and found that CAFs-Si-SDF-1 group had reduced invasion and migration, elevated expression of E-cadherin and lowered N-cadherin. SDF-1 a treatment significantly increased the invasion and migration in A549 cells [37]. Drug-resistant CXCR4-positive cells possess the molecular characteristics of EMT in NSCLC [38]. EMT-related proteins are the regulatory targets for downstream molecules of the SDF-1/CXCR-4 axis [39]. Briefly, SDF-1 secreted by CAFs can promote the invasion and EMT of NSCLC. SDF-1 has high affinity for CXCR4, a specific receptor on the surface of tumor cells [15, 16]. SDF-1 facilitated lung cancer cell proliferation and drug resistance via the CXCR4-mediated pathway $[15,40]$. The activation of SDF-1/CXCR4 axis upregulates IncRNA Xist in rectal cancer [25]. LncRNA Xist is involved in the intimal metastasis and invasion of NSCLC $[26,27]$. RT-qPCR demonstrated that CAFs 
increased the levels of CXCR4 and Xist in A549 or H1299 cells, while silencing SDF-1 in CAFs decreased the expressions of CXCR4 and Xist. Then, we added AMD3100, an inhibitor of CXCR4, to CAFs group. CXCR4 and Xist in CAFs + AMD3100 group were decreased. In contrast, AMD3100 reversed the effect induced by SDF-1 a through decreasing the expression of CXCR-4 [37]. These results suggest that SDF-1 can promote Xist expression through CXCR4.

To study the effect of Xist on NSCLC, si-Xist was delivered to H1299 cells, which were cocultured with CAFs. Xist in CAFs + si-Xist group was also significantly decreased. The invasion and migration of cells in $\mathrm{CAFs}+\mathrm{si}$-Xist group were decreased, E-cadherin was increased and $\mathrm{N}$-cadherin was reduced. Knockdown of IncRNA XIST inhibited EMT and NSCLC cell migration and invasion, and tumor growth in NSCLC xenograft mouse model $[26,27]$. Overall, silencing Xist can reduce the promoting effect of SDF-1 secreted by CAFs on the invasion and metastasis of NSCLC. To further study the molecular mechanism of Xist in NSCLC, we used Starbase to predict the downstream miRNAs. miR-15a-5p is poorly expressed in the serum of NSCLC patients [29]. Then, we predicted the downstream targets of miR-15a-5p. MMP3 affects the migration and invasion of NSCLC[30, 31]. The binding relationships between Xist and miR-15a-5p, and miR-15a-5p and MMP3 were confirmed luciferase-reporter assay and RIP assay. RT-qPCR showed that CAFs reduced miR-15a-5p and increased MMP3, while silencing SDF-1 or AMD3100 or silencing Xist could increase miR-15a-5p and decrease MMP3. In short, IncRNA Xist can target miR-15a-5p to regulate MMP3. To identify the effect of miR-15a-5p on NSCLC, miR-15a-5p mimic was delivered into $\mathrm{H} 1299$ cells. After coculture with CAFs, miR-15a-5p was also upregulated in CAFs + mimic group, the invasion and migration were decreased, E-cadherin was increased and N-cadherin was decreased, and MMP3 was reduced. Overexpression of miR-15a can significantly inhibit A549 cell proliferation, migration and invasion[41, 42]. Collectively, overexpression of miR-15a-5p can reduce the promotion of SDF-1 secreted by CAFs on NSCLC.

Finally, the role of SDF-1 secreted by CAFs in lung cancer metastasis in vivo was studied. CAFs were cocultured with $\mathrm{H} 1299$ cells and injected into mice. H1299 + CAFs treatment showed more metastatic nodules on the lung surface, upregulated Xist, downregulated miR-15a-5p, lowered E-cadherin, and elevated SDF-1, CXCR4, MMP3 and N-cadherin. CXCR4 overexpression leads to abundant and significantly larger tumor lesions in mice [43]. High expression of CXCR4 in primary site and SDF-1 in metastatic lymph nodes was associated with poor overall survival of NSCLC patients[44]. These results suggest that SDF-1 secreted by CAFs can affect Xist/miR-15a-5p/MMP3 through CXCR4 and promote lung metastasis in nude mice.

In summary, we found that SDF-1 secreted by CAFs can promote the invasion and metastasis of NSCLC. The molecular mechanism is that SDF- 1 secreted by CAFs promotes the IncRNA Xist expression by binding to CXCR4, and then inhibits miR-15a-5p and upregulates MMP3 to participate in tumor progression. However, we have not yet studied the role of MMP3 downstream signaling pathway in the invasion and metastasis of NSCLC, and whether other IncRNAs or miRNAs are involved in the progress of SDF-1-mediated NSCLC. Whether IncRNA Xist and miR-15a-5p can become biomarkers for the diagnosis and prognosis of NSCLC and potential therapeutic targets still needs further research. The high rate of 
brain metastasis makes lung cancer the most common type of tumor spreading to the brain [45]. The molecular mechanism of SDF-1 secreted by CAFs involved in brain metastasis of NSCLC remains unclear. CAFs can not only secrete SDF-1 to promote the invasion and metastasis of NSCLC, but also secrete extracellular vesicles into adjacent cells, which are pivotal in tumor occurrence and development[46]. Therefore, we can further study the mechanism of CAFs on NSCLC from exosomes and other aspects.

\section{Declarations}

\section{Funding}

This work was supported by Basic Public Welfare Research Project of Zhejiang Province, China (No.LGF2OH160005) $ه$ Natural Science Foundation of Zhejiang Province, China (No. LY18H160036), and "1022" Talent Training Project of Zhejiang Cancer Hospital.

\section{Competing interests}

The authors declare that they have no competing interests.

\section{Ethics approval}

All procedures were authorized by the academic ethics committee of Cancer Hospital Affiliated to the University of Chinese Academy of Sciences. The experiment was carried out in strict accordance with the guidelines for the management and use of laboratory animals. All the laboratory procedures were used to reduce the pain of the mice.

\section{Consent to participate}

Not applicable.

\section{Availability of data and materials}

All the data generated or analyzed during this study are included in this published article.

\section{Authors' contributions}

LW, HZ contributed to the study concepts, study design, and definition of intellectual content; LW,WC contributed to the literature research; $\mathrm{LW}, \mathrm{XL}$ contributed to the manuscript preparation and $\mathrm{HZ}$ contributed to the manuscript editing and review; LW, WC, XL contributed to the experimental studies and data acquisition; All authors read and approved the final manuscript.

\section{References}

1. Spella M, Stathopoulos GT: Immune Resistance in Lung Adenocarcinoma.Cancers (Basel) 2021, 13. 
2. Pirlog R, Cismaru A, Nutu A, Berindan-Neagoe I: Field Cancerization in NSCLC: A New Perspective on MicroRNAs in Macrophage Polarization.Int J Mol Sci 2021, 22.

3. Ye Z, Huang Y, Ke J, Zhu X, Leng S, Luo H: Breakthrough in targeted therapy for non-small cell lung cancer.Biomed Pharmacother 2021, 133:111079.

4. Chatziandreou I, Tsioli P, Sakellariou S, Mourkioti I, Giannopoulou I, Levidou G, Korkolopoulou P, Patsouris E, Saetta AA: Comprehensive Molecular Analysis of NSCLC; Clinicopathological Associations.PLoS One 2015, 10:e0133859.

5. Ortega-Franco A, Calvo V, Franco F, Provencio M, Califano R: Integrating immune checkpoint inhibitors and targeted therapies in the treatment of early stage non-small cell lung cancer: a narrative review. Trans/ Lung Cancer Res 2020, 9:2656-2673.

6. Schoenmaekers J, Paats MS, Dingemans AC, Hendriks LEL: Central nervous system metastases and oligoprogression during treatment with tyrosine kinase inhibitors in oncogene-addicted non-small cell lung cancer: how to treat and when? Trans/ Lung Cancer Res 2020, 9:2599-2617.

7. Zang J, Hu Y, Xu X, Ni J, Yan D, Liu S, He J, Xue J, Wu J, Feng J: Elevated serum levels of vascular endothelial growth factor predict a poor prognosis of platinum-based chemotherapy in non-small cell lung cancer. Onco Targets Ther 2017, 10:409-415.

8. Nokin MJ, Ambrogio C, Nadal E, Santamaria D: Targeting Infrequent Driver Alterations in Non-Small Cell Lung Cancer.Trends Cancer 2020.

9. She Q, Hu S, Pu X, Guo Q, Mou C, Yang C: The effect of hepatocellular carcinoma-associated fibroblasts on hepatoma vasculogenic mimicry.Am J Cancer Res 2020, 10:4198-4210.

10. Shi Y, Sun L, Zhang R, Hu Y, Wu Y, Dong X, Dong D, Chen C, Geng Z, Li E, Fan Y: Thrombospondin 4/integrin alpha2/HSF1 axis promotes proliferation and cancer stem-like traits of gallbladder cancer by enhancing reciprocal crosstalk between cancer-associated fibroblasts and tumor cells.J Exp Clin Cancer Res 2021, 40:14.

11. Baker AT, Abuwarwar MH, Poly L, Wilkins S, Fletcher AL: Cancer-Associated Fibroblasts and T Cells: From Mechanisms to Outcomes.J Immunol 2021, 206:310-320.

12. Shintani Y, Abulaiti A, Kimura T, Funaki S, Nakagiri T, Inoue M, Sawabata N, Minami M, Morii E, Okumura M: Pulmonary fibroblasts induce epithelial mesenchymal transition and some characteristics of stem cells in non-small cell lung cancer.Ann Thorac Surg 2013, 96:425-433.

13. Wei T, Song J, Liang K, Li L, Mo X, Huang Z, Chen G, Mao N, Yang J: Identification of a novel therapeutic candidate, NRK, in primary cancer-associated fibroblasts of lung adenocarcinoma microenvironment.J Cancer Res Clin Oncol 2021, 147:1049-1064.

14. Alguacil-Nunez C, Ferrer-Ortiz I, Garcia-Verdu E, Lopez-Pirez P, Llorente-Cortijo IM, Sainz B, Jr.: Current perspectives on the crosstalk between lung cancer stem cells and cancer-associated fibroblasts. Crit Rev Oncol Hematol 2018, 125:102-110.

15. Li J, Guan J, Long X, Wang Y, Xiang X: mir-1-mediated paracrine effect of cancer-associated fibroblasts on lung cancer cell proliferation and chemoresistance.Oncol Rep 2016, 35:3523-3531. 
16. Zhou Y, Cao HB, Li WJ, Zhao L: The CXCL12 (SDF-1)/CXCR4 chemokine axis: Oncogenic properties, molecular targeting, and synthetic and natural product CXCR4 inhibitors for cancer therapy.Chin $J$ Nat Med 2018, 16:801-810.

17. Liu H, Cheng Q, Xu DS, Wang W, Fang Z, Xue DD, Zheng Y, Chang AH, Lei YJ: Overexpression of CXCR7 accelerates tumor growth and metastasis of lung cancer cells.Respir Res 2020, 21:287.

18. Zhou W, Guo S, Liu M, Burow ME, Wang G: Targeting CXCL12/CXCR4 Axis in Tumor Immunotherapy.Curr Med Chem 2019, 26:3026-3041.

19. Wu Y, Shen QW, Niu YX, Chen XY, Liu HW, Shen XY: LncNORAD interference inhibits tumor growth and lung cancer cell proliferation, invasion and migration by down-regulating CXCR4 to suppress RhoAVROCK signaling pathway.Eur Rev Med Pharmacol Sci 2020, 24:5446-5455.

20. Livak KJ, Schmittgen TD: Analysis of relative gene expression data using real-time quantitative PCR and the 2(-Delta Delta C(T)) Method.Methods 2001, 25:402-408.

21. Arbour KC, Riely GJ: Systemic Therapy for Locally Advanced and Metastatic Non-Small Cell Lung Cancer: A Review.JAMA 2019, 322:764-774.

22. Kong X, Zhao Y, Li X, Tao Z, Hou M, Ma H: Overexpression of HIF-2alpha-Dependent NEAT1 Promotes the Progression of Non-Small Cell Lung Cancer through miR-101-3p/SOX9/Wnt/beta-Catenin Signal Pathway.Cell Physiol Biochem 2019, 52:368-381.

23. Mahmood MQ, Ward C, Muller HK, Sohal SS, Walters EH: Epithelial mesenchymal transition (EMT) and non-small cell lung cancer (NSCLC): a mutual association with airway disease.Med Oncol 2017, $34: 45$.

24. Yuan X, Wu H, Han N, Xu H, Chu Q, Yu S, Chen Y, Wu K: Notch signaling and EMT in non-small cell lung cancer: biological significance and therapeutic application.J Hematol Oncol 2014, 7:87.

25. Yu X, Wang D, Wang X, Sun S, Zhang Y, Wang S, Miao R, Xu X, Qu X: CXCL12/CXCR4 promotes inflammation-driven colorectal cancer progression through activation of RhoA signaling by sponging miR-133a-3p.J Exp Clin Cancer Res 2019, 38:32.

26. Li C, Wan L, Liu Z, Xu G, Wang S, Su Z, Zhang Y, Zhang C, Liu X, Lei Z, Zhang HT: Long non-coding RNA XIST promotes TGF-beta-induced epithelial-mesenchymal transition by regulating miR-367/141ZEB2 axis in non-small-cell lung cancer.Cancer Lett 2018, 418:185-195.

27. Zhang YL, Li XB, Hou YX, Fang NZ, You JC, Zhou QH: The IncRNA XIST exhibits oncogenic properties via regulation of miR-449a and Bcl-2 in human non-small cell lung cancer.Acta Pharmacol Sin 2017, 38:371-381.

28. Li JH, Liu S, Zhou H, Qu LH, Yang JH: starBase v2.0: decoding miRNA-ceRNA, miRNA-ncRNA and protein-RNA interaction networks from large-scale CLIP-Seq data.Nucleic Acids Res 2014, 42:D92-97.

29. Kumar S, Sharawat SK, Ali A, Gaur V, Malik PS, Kumar S, Mohan A, Guleria R: Identification of differentially expressed circulating serum microRNA for the diagnosis and prognosis of Indian nonsmall cell lung cancer patients.Curr Probl Cancer 2020, 44:100540.

30. Jiang J, Ren HY, Geng GJ, Mi YJ, Liu Y, Li N, Yang SY, Shen DY: Oncogenic activity of insulin in the development of non-small cell lung carcinoma.Oncol Lett 2018, 15:447-452. 
31. Jin G, Miao R, Hu Z, Xu L, Huang X, Chen Y, Tian T, Wei Q, Boffetta P, Shen H: Putative functional polymorphisms of MMP9 predict survival of NSCLC in a Chinese population.Int J Cancer 2009, 124:2172-2178.

32. Ba P, Xu M, Yu M, Li L, Duan X, Lv S, Fu G, Yang J, Yang P, Yang C, Sun Q: Curcumin suppresses the proliferation and tumorigenicity of $\mathrm{Cal} 27$ by modulating cancer-associated fibroblasts of TSCC.Oral Dis 2020, 26:1375-1383.

33. Kovacs D, Igaz N, Marton A, Ronavari A, Belteky P, Bodai L, Spengler G, Tiszlavicz L, Razga Z, Hegyi P, et al: Core-shell nanoparticles suppress metastasis and modify the tumour-supportive activity of cancer-associated fibroblasts.J Nanobiotechnology 2020, 18:18.

34. Zeng Y, Du Q, Zhang Z, Ma J, Han L, Wang Y, Yang L, Tao N, Qin Z: Curcumin promotes cancerassociated fibroblasts apoptosis via ROS-mediated endoplasmic reticulum stress.Arch Biochem Biophys 2020, 694:108613.

35. Luo M, Luo Y, Mao N, Huang G, Teng C, Wang H, Wu J, Liao X, Yang J: Cancer-Associated Fibroblasts Accelerate Malignant Progression of Non-Small Cell Lung Cancer via Connexin 43-Formed Unidirectional Gap Junctional Intercellular Communication.Cell Physiol Biochem 2018, 51:315-336.

36. Rodriguez-Lara V, Pena-Mirabal E, Baez-Saldana R, Esparza-Silva AL, Garcia-Zepeda E, Cerbon Cervantes MA, Diaz D, Fortoul TI: Estrogen receptor beta and CXCR4/CXCL12 expression: differences by sex and hormonal status in lung adenocarcinoma.Arch Med Res 2014, 45:158-169.

37. Xia R, Xu G, Huang Y, Sheng X, Xu X, Lu H: Hesperidin suppresses the migration and invasion of nonsmall cell lung cancer cells by inhibiting the SDF-1/CXCR-4 pathway.Life Sci 2018, 201:111-120.

38. Yin H, Wang Y, Chen W, Zhong S, Liu Z, Zhao J: Drug-resistant CXCR4-positive cells have the molecular characteristics of EMT in NSCLC.Gene 2016, 594:23-29.

39. Grundker C, Bauerschmitz G, Knapp J, Schmidt E, Olbrich T, Emons G: Inhibition of SDF-1/CXCR4induced epithelial-mesenchymal transition by kisspeptin-10.Breast Cancer Res Treat 2015, 152:4150.

40. Sterlacci W, Saker S, Huber B, Fiegl M, Tzankov A: Expression of the CXCR4 ligand SDF-1/CXCL12 is prognostically important for adenocarcinoma and large cell carcinoma of the lung. Virchows Arch 2016, 468:463-471.

41. Guo S, Li M, Li J, Lv Y: Inhibition mechanism of lung cancer cell metastasis through targeted regulation of Smad3 by miR-15a.Oncol Lett 2020, 19:1516-1522.

42. He J: Knocking down MiR-15a expression promotes the occurrence and development and induces the EMT of NSCLC cells in vitro.Saudi J Biol Sci 2017, 24:1859-1865.

43. Jager B, Klatt D, Plappert L, Golpon H, Lienenklaus S, Barbosa PD, Schambach A, Prasse A: CXCR4/MIF axis amplifies tumor growth and epithelial-mesenchymal interaction in non-small cell lung cancer.Cell Signal 2020, 73:109672.

44. Katsura M, Shoji F, Okamoto T, Shimamatsu S, Hirai F, Toyokawa G, Morodomi Y, Tagawa T, Oda Y, Maehara Y: Correlation between CXCR4/CXCR7/CXCL12 chemokine axis expression and prognosis in lymph-node-positive lung cancer patients.Cancer Sci 2018, 109:154-165. 
45. Cavallaro S: CXCR4/CXCL12 in non-small-cell lung cancer metastasis to the brain.Int J Mol Sci 2013, 14:1713-1727.

46. Yang F, Yan Y, Yang Y, Hong X, Wang M, Yang Z, Liu B, Ye L: MiR-210 in exosomes derived from CAFs promotes non-small cell lung cancer migration and invasion through PTEN/PI3K/AKT pathway.Cell Signal 2020, 73:109675.

\section{Figures}

A

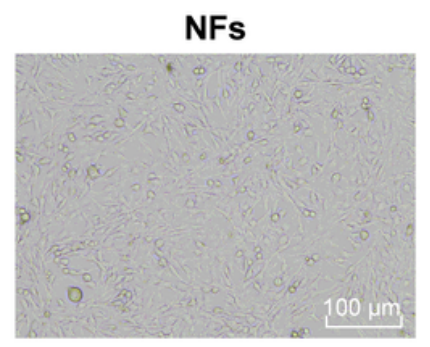

CAFs

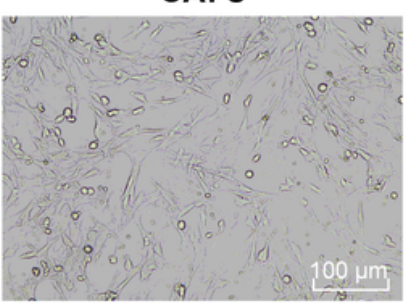

B
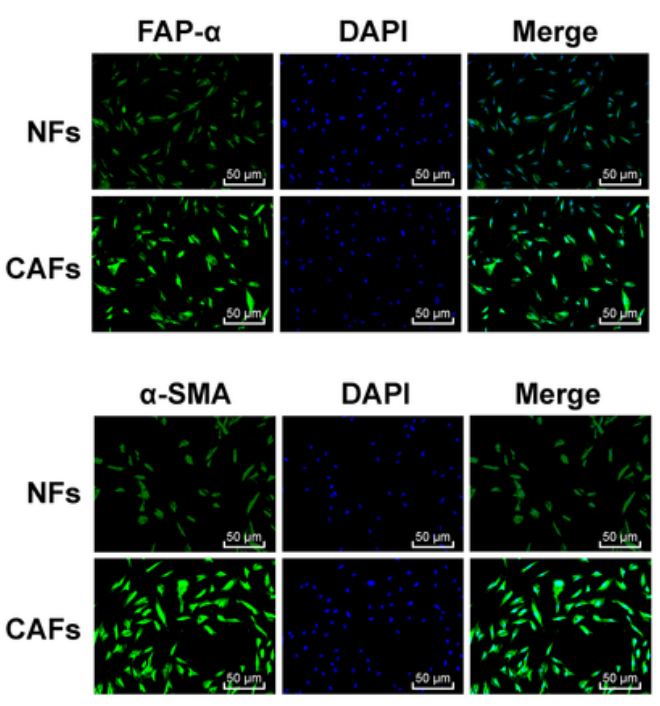

C

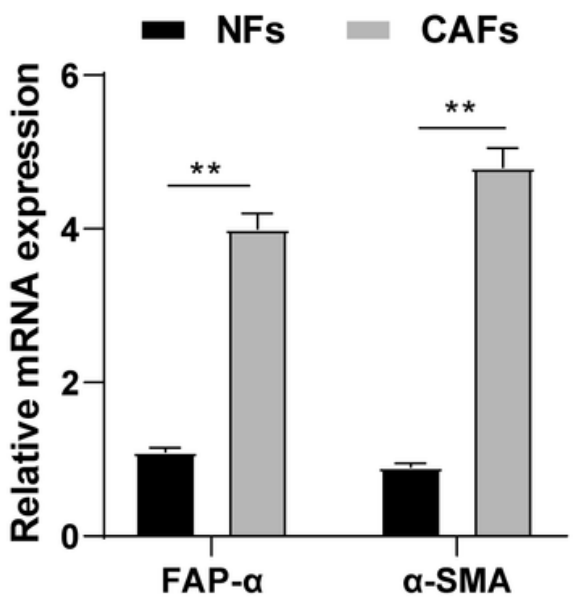

Figure 1

Isolation and culture of CAFs and NFs. (A) The morphology of CAFs and NFs was observed under microscope; (B) immunofluorescence staining of FAP-a and a-SMA; (C) RT-qPCR was used to detect the mRNA expression of FAP- $a$ and a-SMA; cell experiment was carried out three times independently, the data were expressed as mean \pm standard deviation; the data in panel $C$ were analyzed by two-way ANOVA, followed by Sidak's multiple comparisons test, **, $p<0.01$. 
A
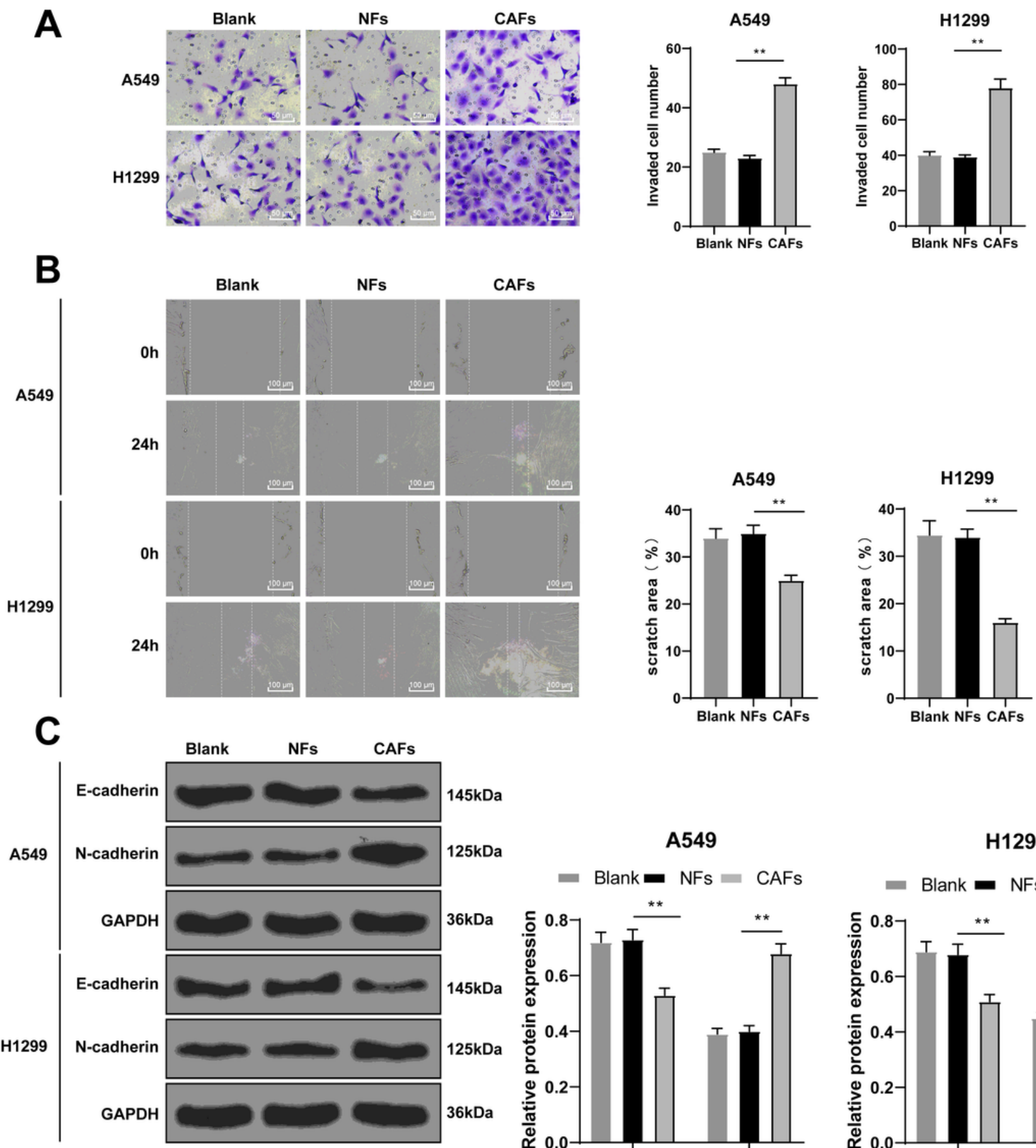

A549

- Blank $=\mathrm{NFs}=\mathrm{CAFs}$

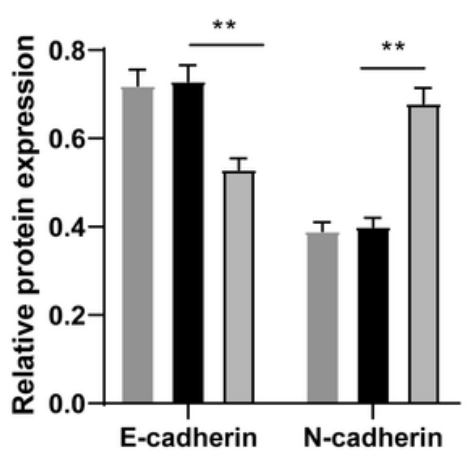

H1299

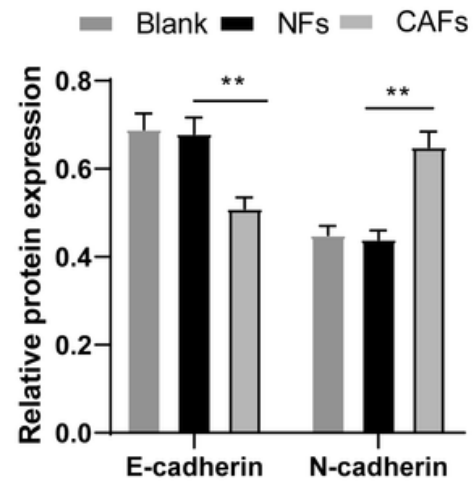

Figure 2

CAFs promote invasion and metastasis of NSCLC. Human NSCLC cells A549/H1299 were cocultured with CAFs or NFs at the ratio of 5:1. (A) The invasion ability was detected by Transwell assay; (B) The migration ability was detected by scratch test; (C) Protein levels of E-cadherin and N-cadherin were detected by Western blot; cell experiment was carried out three times independently, the data were expressed as mean \pm standard deviation; the data in panels A/B were analyzed by two-way ANOVA, and 
data in panel $\mathrm{C}$ were analyzed by one-way ANOVA, followed by Tukey's multiple comparisons test, $* \star, p<$ 0.01 .

A
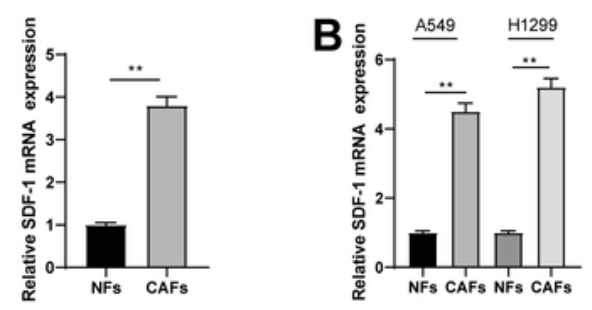

E

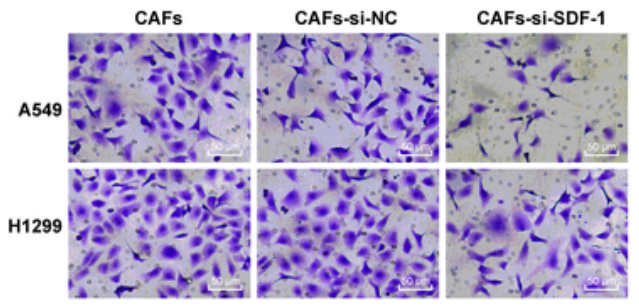

$\mathbf{F}$

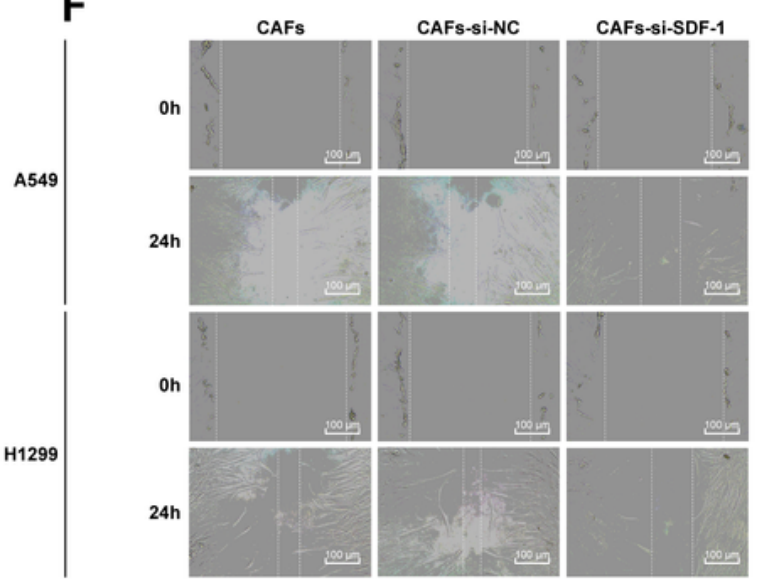

G

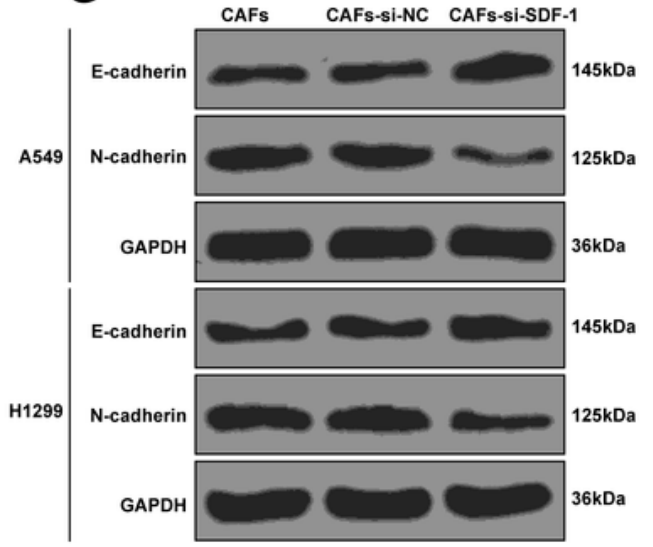

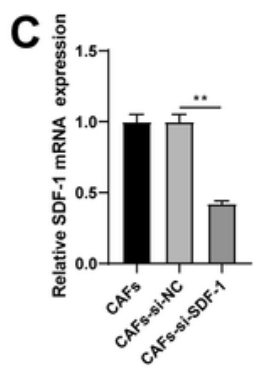

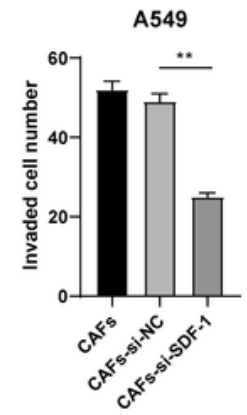

A549
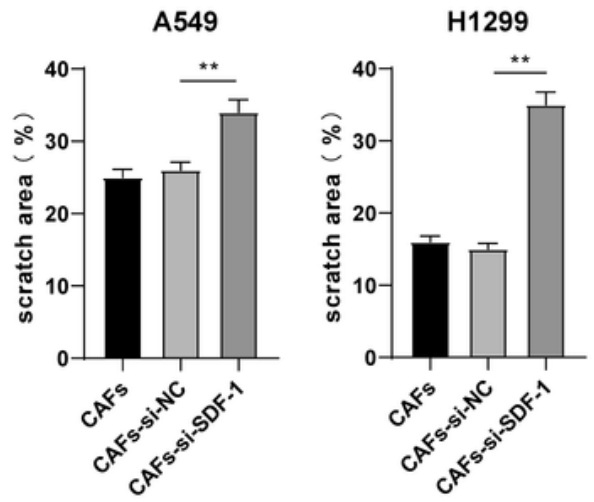

\section{Figure 3}
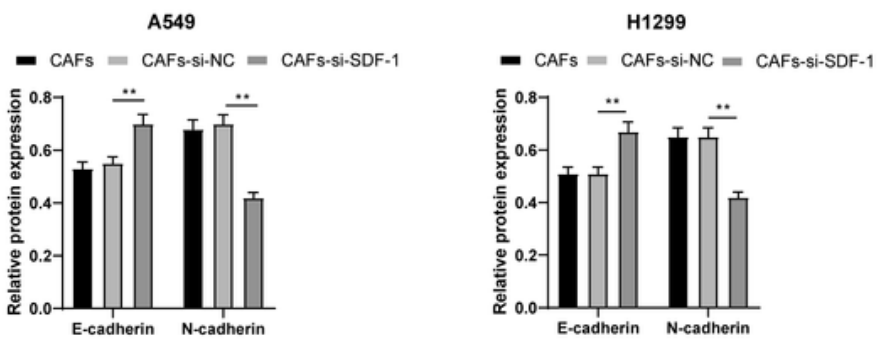

SDF-1 secreted by CAFs promotes invasion and metastasis of NSCLC. (A) RT-qPCR was used to detect the expression of SDF-1 in CAFs; (B) RT-qPCR was used to detect the expression of SDF-1 after CAFs were cocultured with A549 or H1299 cells; si-SDF-1 was transfected into CAFs, with si-NC as control, and then 
cocultured with A549 or H1299; (C) RT-qPCR was used to detect the transfection efficiency of si-SDF-1 in CAFs; (D) RT-qPCR was used to detect the expression of SDF-1 after CAFs were cocultured with A549 or H1299 cells; (E) The invasion ability was detected by Transwell assay; $(F)$ The migration was detected by scratch test; (G) Protein levels of E-cadherin and N-cadherin were detected by Western blot; cell experiment was carried out three times independently, the data were expressed as mean \pm standard deviation; the data in panels $A / B$ were analyzed by $t$ test, data in panels $C-F$ were analyzed by one-way ANOVA, and data in panel $G$ were analyzed by two-way ANOVA, followed by Tukey's multiple comparisons test, $* *, p<0.01$.
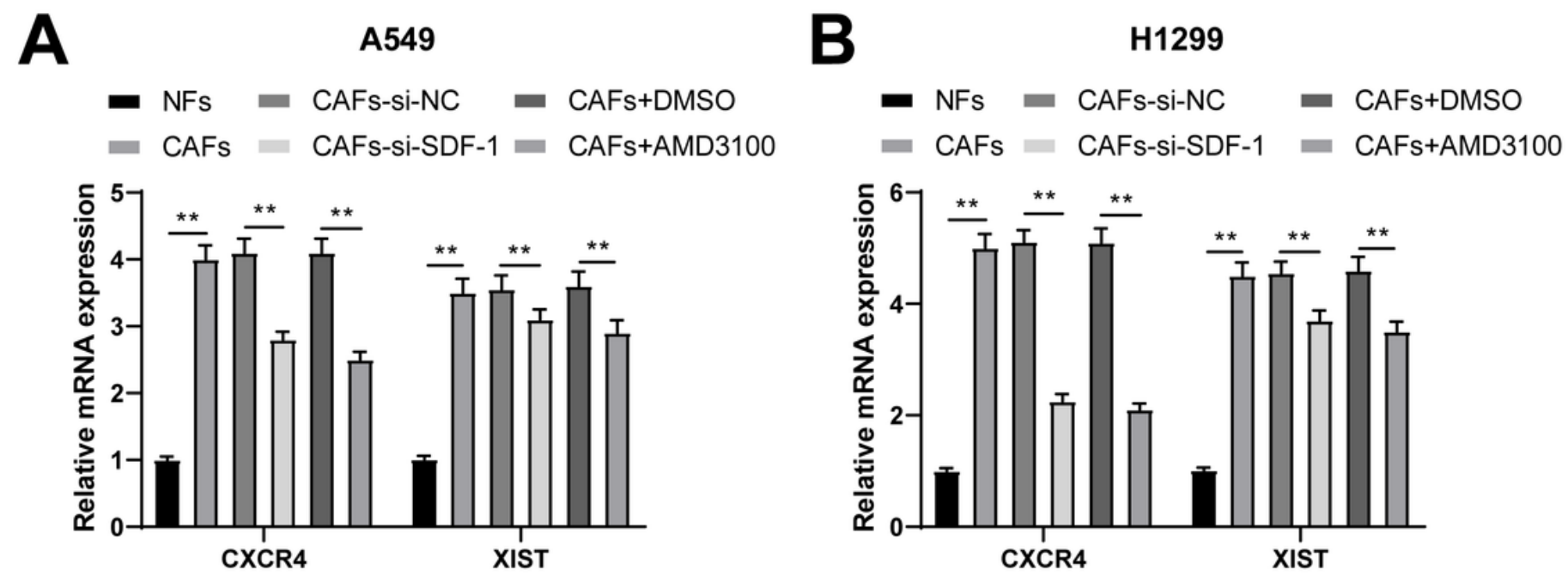

Figure 4

SDF-1 promotes IncRNA Xist expression through CXCR4. (A) RT-qPCR was used to detect the mRNA expression of CXCR and Xist in A549 cells; (B) RT-qPCR was used to detect the mRNA expression of CXCR4 and Xist in H1299 cells; cell experiment was carried out three independently, and the data were expressed as mean \pm standard deviation; the data in panels were analyzed by two-way ANOVA, followed by Tukey's multiple comparisons test, $* \star, p<0.01$. 
A

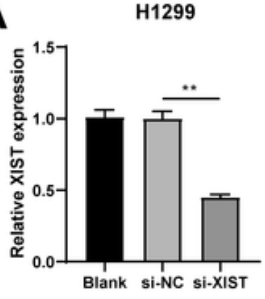

B

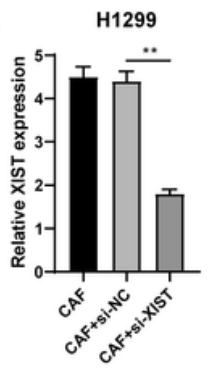

D

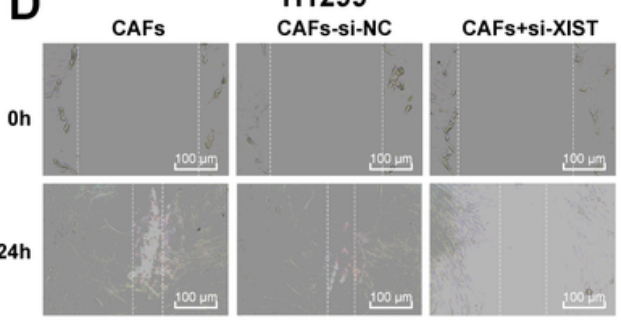

C
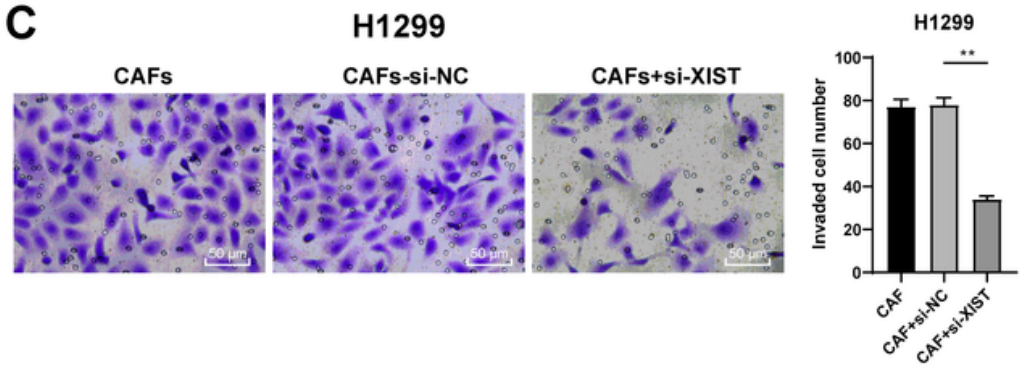
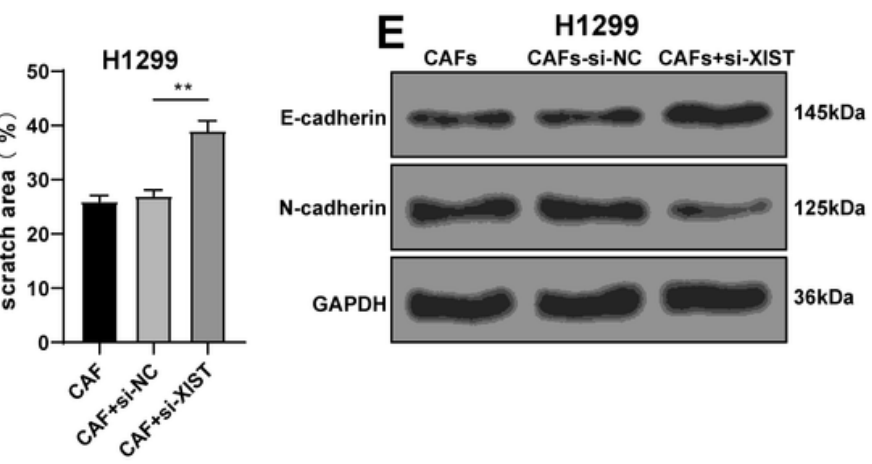

H1299

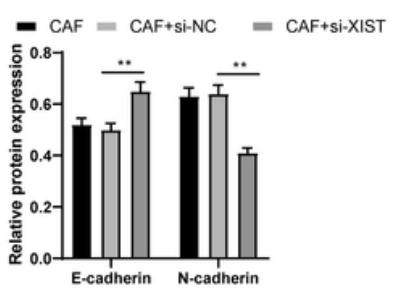

\section{Figure 5}

Silencing Xist can reduce the promoting effect of SDF-1 secreted by CAFs on the invasion and metastasis of NSCLC. si-Xist was transfected into $\mathrm{H} 1299$ cells then cocultured with CAFs, with si-NC as control. (A) RT-qPCR was used to detect the expression of si-Xist in H1299 cells; (B) RT-qPCR was used to detect the expression of Xist in H1299 cells cocultured with CAFs; (C) The invasion was detected by Transwell assay; (D) The migration ability was detected by scratch test; (E) Protein levels of E-cadherin and Ncadherin were detected by Western blot; cell experiment was carried out three times independently, the data were expressed as mean \pm standard deviation; the data in panels A-D were analyzed by one-way ANOVA, and data in panel E were analyzed by two-way ANOVA, followed by Tukey's multiple comparisons test, $* \star, p<0.01$. 


\begin{tabular}{|c|c|c|c|c|c|c|c|c|}
\hline miRNA & & GenelD & GeneName & GeneType & & TargetSite & & Alignment \\
\hline hsa-miR-15a-5p & $\uparrow$ & ENSG00000229807 & XIST & lincRNA & $\uparrow$ & chrX:73071755-73071776[-] & $\uparrow$ & 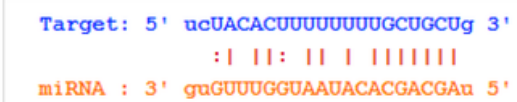 \\
\hline
\end{tabular}

B
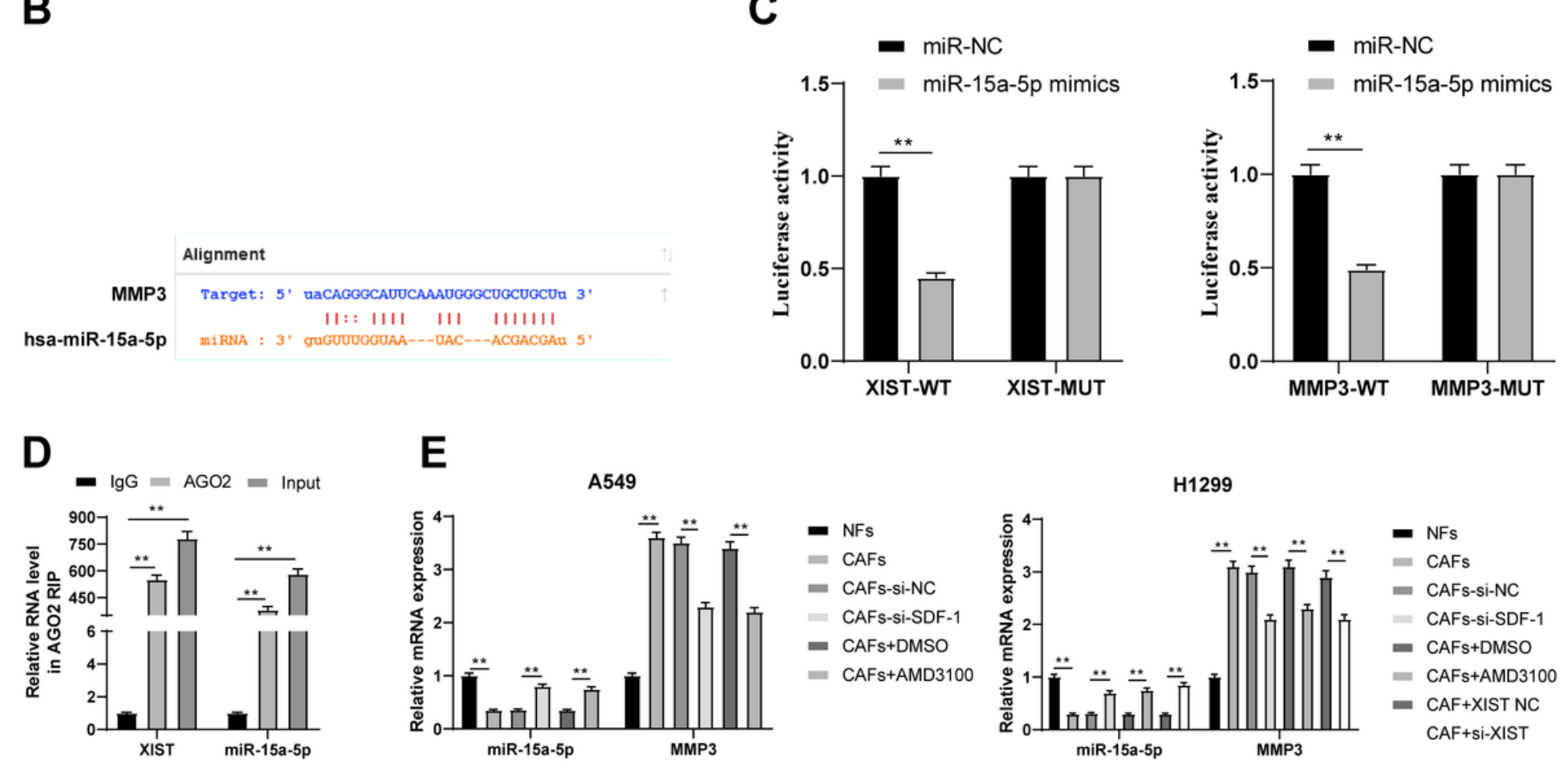

\section{Figure 6}

LncRNA Xist can target miR-15a-5p to regulate MMP3. (A-B) Binding relationships between IncRNA Xist and miR-15a-5p, and miR-15a-5p and MMP3 were predicted by Starbase; (C) the targeting relationships were verified by dual-luciferase assay; (D) Relationship between IncRNA Xist and miR-15a-5p was detected by RIP assay; (E) Expression of miR-15a-5p and MMP3 was detected by RT-qPCR; cell experiment was carried out three times independently, the data were expressed as mean \pm standard deviation; the data in panels C-E were analyzed by two-way ANOVA, followed by Tukey's multiple comparisons test, $* \star, p<0.01$. 

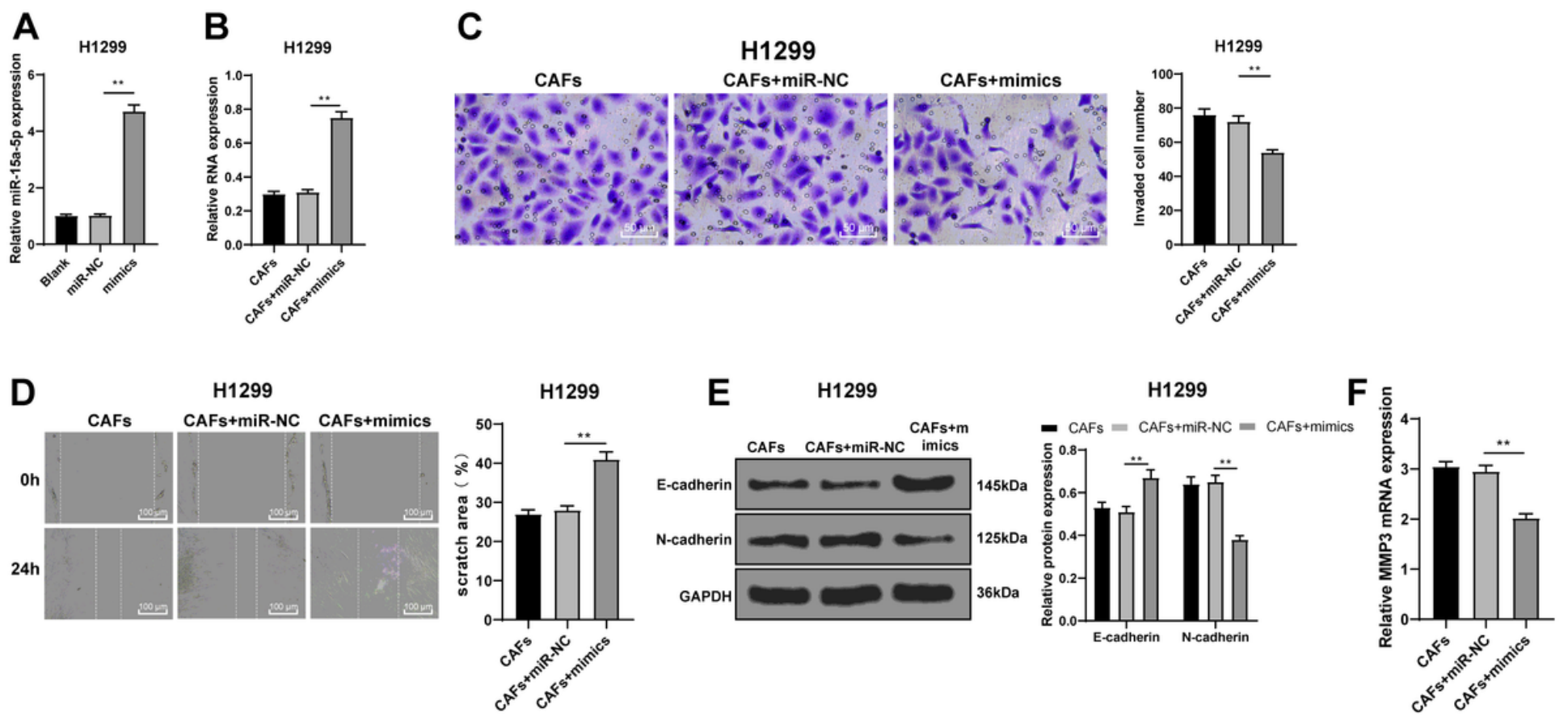

Figure 7

Overexpression of miR-15a-5p can reduce the promoting effect of SDF-1 secreted by CAFs on NSCLC. miR-15a-5p mimic were transfected into $\mathrm{H} 1299$ cells, which were cocultured with CAFs. (A) RT-qPCR was used to detect the expression of miR-15a-5p in H1299; (B) RT-qPCR was used to detect the expression of miR-15a-5p after coculture of miR-15a-5p overexpressing H1299 cells and CAFs; (C) The invasion was detected by Transwell assay; (D) The migration ability was detected by scratch test; (E) Protein levels of E-cadherin and N-cadherin were detected by Western blot; (F) RT-qPCR was used to detect the expression of MMP3 in H1299 cells; cell experiment was carried out three times independently, the data were expressed as mean \pm standard deviation; the data in panels A-D and F were analyzed by one-way ANOVA, and data in panel E were analyzed by two-way ANOVA, followed by Tukey's multiple comparisons test, **, $p<0.01$. 
A

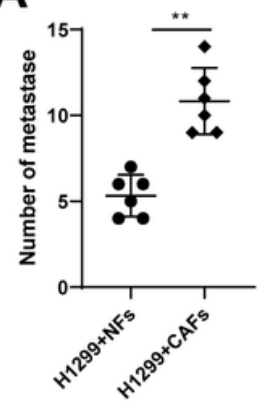

B

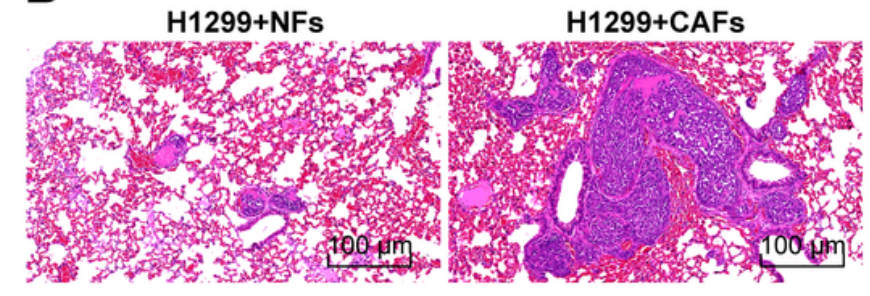

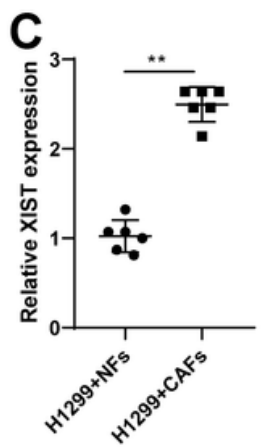

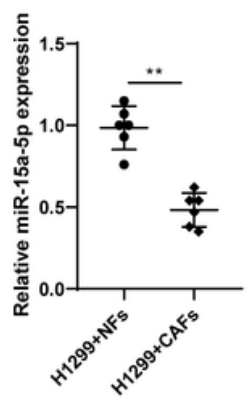

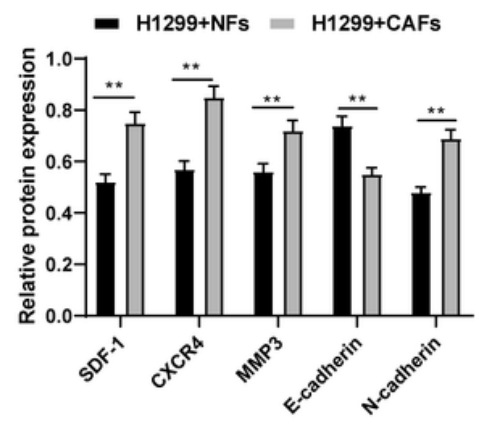

\section{Figure 8}

SDF-1 secreted by CAFs can affect Xist/miR-15a-5p/MMP3 through CXCR4 and promote lung metastasis in nude mice. $\mathrm{H} 1299$ cells, CAFs and NFs were cocultured and injected into mice via tail vein. The mice were killed after 4 weeks. (A) The number of metastatic nodules in lung tissue; (B) HE staining of metastatic nodules in typical lung tissue; (C) The expressions of Xist and miR-15a-5p in lung tissue were detected by RT-qPCR; (D) SDF-1, CXCR4, MMP3, E-cadherin, N-cadherin protein levels were detected by Western blot; $\mathrm{N}=6$, the data were expressed as mean \pm standard deviation; the data in panels $\mathrm{A}$ and $\mathrm{C}$ were analyzed by $t$ test, and data in panel $D$ were analyzed by two-way ANOVA, followed by Tukey's multiple comparisons test, $* *, p<0.01$. 